\title{
Design of Endoscopes for Monitoring Water-Cooled Divertor in W7-X
}

\author{
Joris Fellinger $^{a^{*}}$, Uwe Lippmann ${ }^{\mathrm{b}}$, Henry Greve $^{\mathrm{a}}$, Mohamad Alhashimi ${ }^{\mathrm{a}}$, Mathias Schülke ${ }^{\mathrm{a}}$, Simppa \\ Äkaslompolo ${ }^{a}$, Peter Drewelow ${ }^{a}$, Marcin Jakubowski ${ }^{a}$, Ralf König ${ }^{\mathrm{a}}$, Axel Lorenz ${ }^{\mathrm{a}}$ and the W7-X team \\ ${ }^{a}$ Max Planck Institute for Plasmaphysik, EUROATOM Association, Wendelsteinstrasse 1, 17489, \\ Greifswald, Germany \\ ${ }^{b}$ Fraunhofer Institute for Applied Optics and Precision Engineering IOF, Albert-Einstein-Strasse 7, \\ 07745 Jena, Germany
}

\begin{abstract}
The modular stellarator Wendelstein 7-X (W7-X) in Greifswald (Germany) started operation in 2015 with short pulse limiter plasmas and continued with pulsed divertor plasmas in 2017-2018. In 2021, the next operation phase (OP) OP2 will start after installation of 10 water-cooled CFC armored divertors, allowing for steady state operation.

Since divertor heat loads are very sensitive to plasma parameters, each water-cooled divertor needs to be monitored to interrupt or adapt plasma operation once overload is detected. For that purpose ten endoscopes are planned: two in module 3 and eight more in a different type of port in the other modules. The infrared (IR) radiation from the plasma facing surface as well as the plasma edge radiation in the visible (VIS) range is captured through a pinhole in a water-cooled plasma facing head and transmitted to the rear side outside the vacuum where the light is split and captured by an IR and VIS camera. The design challenge is to reach a high-resolution image of the entire target while capturing a large field of view (FOV) of 120 degrees.

In this paper, the design and assembly strategy is presented, including the assessment of the optical, thermomechanical and hydraulic performance.
\end{abstract}

Keywords: Wendelstein 7-X, Divertor Thermography, Endoscopes, Infrared, Optical Design, ECRH Stray Radiation

\section{Introduction}

Wendelstein 7-X, the largest modular stellarator in the world, is currently being upgraded with ten identical water-cooled divertors, designed to withstand $10 \mathrm{MW} / \mathrm{m}^{2}$ in steady state operation (max. 30 minutes due to cooling basin restrictions). To protect the divertor from damage, each divertor has to be monitored. During the short pulse operation phases (OP) of 2015-2018 (OP1.1 and OP1.2) uncooled immersion tube systems and uncooled endoscope were used to monitor the divertor [1]-[2]. In addition, an uncooled monitoring system was developed at IPP to obtain a high-resolution view of the so-called scraper elements [3]. For long pulse operation, these systems have to be replaced by water-cooled endoscope systems.

There are two types of endoscopes: By default, the endoscopes will be installed in the so-called AEF ports (8x) except those in module $3(2 \mathrm{x})$ which have to be installed in the AEA ports to avoid collisions with the ICRH antennas. The view through the pinhole onto the divertor is shown in Fig. 1.

This paper presents the design and assessment of the performance and W7-X compatibility of the AEA endoscopes (those in the AEA ports). The AEF endoscope will be a copy with slightly different shape of most components resulting from a different viewing angle. Since a part of the divertor, i.e. vertical target module 1 (TM1v) cannot be seen from the AEA port, a simple IR observation system outside the AEK ports is planned in module 3 a with straight view to this area.

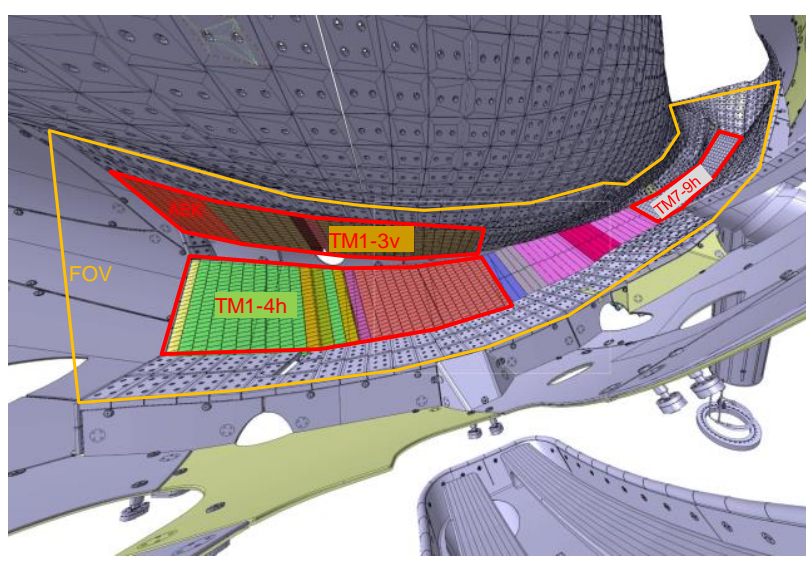

Fig. 1: View from the pinhole of the AEF endoscope onto the divertor: High resolution is required for the red marked areas, the orange area defines the field of view.

In the next chapter, the basic features of the endoscope design are outlined. In chapter 3, the optical design using a Zemax model is described in more detail. With the model the diffraction and aberration-based limits of the optical performance are balanced. In chapter 4, thermal and mechanical analyses are presented. The distribution of ECRH stray radiation inside the endoscope is determined first and then the thermal and mechanical response is evaluated. The impact of thermal deformations on the optical performance is also assessed. In chapter 5, the tolerance assessment of the optical design is presented. The tolerances and compensators depend on the manufacturing, verification and assembly strategy which is also explained. In chapter 6 , the design of the water-cooled head is presented and the corresponding thermal and hydraulic calculations are compared with 
tests on a full-scale prototype. Finally, in chapter 7 an outlook in the assembly, testing program and installation onto $\mathrm{W} 7-\mathrm{X}$ is given.

\section{Basic design}

The endoscope consists of a vacuum compatible plugin inside the port and an optical box attached to the outside of the vacuum flange, see Fig. 2.

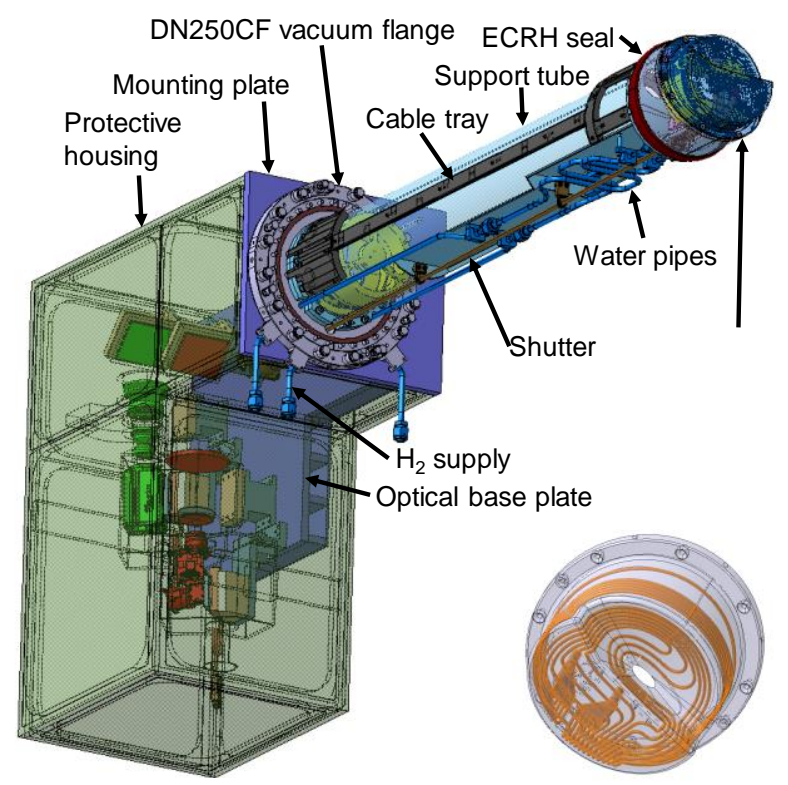

Fig. 2: Lay out of the endoscope and transparent view of the water-cooled head with the cooling channels (insert)

The infrared radiation from the divertor surface and the visible light from the plasma edge passes through a pinhole in a protective water-cooled head at the plasma side of the plug-in. Inside the plug-in, the light is reflected by two front mirrors and beamed towards the rear end of the plug-in. A telescope system collects the light and passes it through a flat sapphire vacuum window. Outside the vacuum, the light is split into an infrared (IR) channel at 3.6-4.5 $\mu \mathrm{m}$ and a visible light (VIS) channel at 350-900 $\mathrm{nm}$. In each channel, the light passes a corrector lens, one or two fold mirrors and an objective lens before it is captured by a camera. The IR camera is based on the SCD Hercules detector, a helium-cooled InSb sensor with $1280 \times 1028$ pixels of $15 \mu \mathrm{m}$. The VIS camera is the PCO.Edge 5.5 CMOS camera with $2560 \times 2160$ pixels of $6.5 \mu \mathrm{m}$. In the objective lens of the VIS channel, a filter wheel allows for five different narrow band pass filters to distinguish between species of plasma edge radiation, see Fig. 3. The water-cooled head with the pinhole is a 3Dprinted part of stainless steel EN 1.4404. It allows for a nearly full geometric freedom. The complex $3 \mathrm{D}$ shape is adapted to fit into the reserved cutout in the plasma facing wall in the plasma vessel, while not obstructing the lines of sight from the divertor to the pinhole and inside the head from pinhole via the two front mirrors towards the rear of the plugin. Six parallel cooling channels are designed within the $8 \mathrm{~mm}$ wall thickness, see Fig. 2 .

A rotating pneumatic shutter with straight axis can close the pinhole during a glow discharge or boronization cycle of W7-X to protect the optics inside the plug-in against pollution and arcing. Nevertheless, the reflectivity of the front mirrors behind the pinhole is expected to deteriorate over time due to contamination by neutrals entering the pinhole during plasma operation. To clean the mirrors, a $200 \mathrm{~W}$ heater and two Pt1000 sensors are inserted in each mirror to enable a controlled heating cycle. The mirrors are made of RSA905, a kind of aluminum alloy, which is stress-released at $380{ }^{\circ} \mathrm{C}$, to allow for a heating cycle up to this temperature without irreversible deformations by the release of internal stress. The high thermal conductivity of RSA905 together with the insulated mirror supports ensures that thermal gradients remain small. To improve the cleanability of the mirrors, a permanent $\mathrm{H}_{2}$ overpressure is planned in one endoscope, with the aim to increase the hydrogen content of the hydrocarbon contamination layers on the mirrors, which is expected to be more easily removed during the heating cycle. The $\mathrm{H}_{2}$ pressure inside the plug-in is monitored with a cold cathode. A passive needle valve in the supply line fixes the maximum $\mathrm{H}_{2}$ flow to such low level that the concentration inside the plug-in is always below the inflammability limit, i.e. the design is inherently safe.

The pinhole closure on the shutter is also used as calibration source for the cameras that can be used in between plasma discharges. The camera facing side of the closure consists of a copper plate that can be rapidly heated by a $50 \mathrm{~W}$ heater with a Pt1000 sensor $(\sim 50 \mathrm{~K} / \mathrm{s})$. To calibrate the IR camera, the closure is heated up to $400{ }^{\circ} \mathrm{C}$ and the IR radiation from the copper plate is captured by the IR camera. To calibrate the VIS camera, a white LED light source in the optical box shines via the optical path onto the copper plate and the reflected light is captured by the VIS camera.

\section{Optical design}

The objective of the optical design is twofold: 1) The field of view (FOV) that is imaged onto the sensor must encompass the entire plasma facing surface of the divertor and baffles. 2) The resolution of the highly loaded areas of the divertor, i.e. the horizontal target module 1 to 4 and
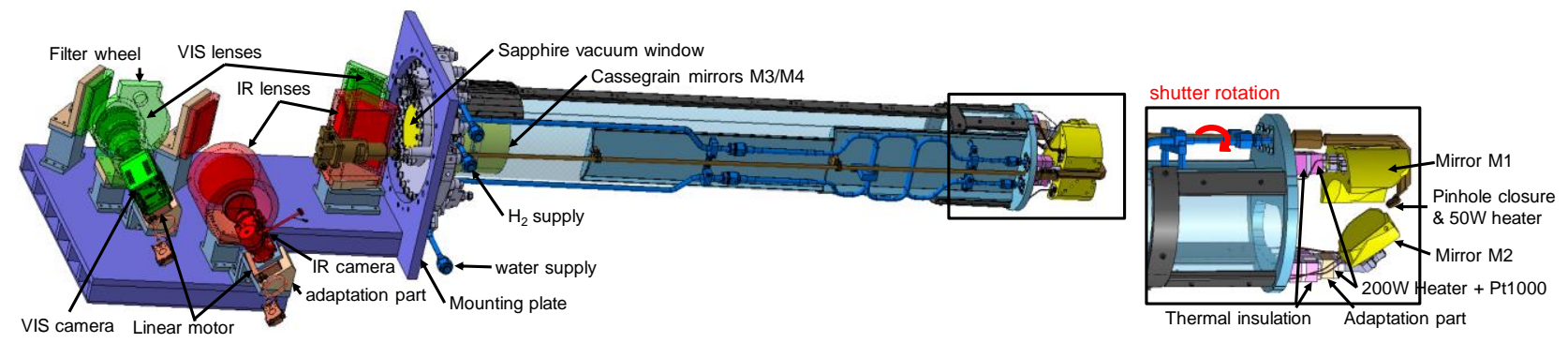

Fig. 3: View into optical box and water-cooled head to show the optical components: IR channel in red and VIS channel in green 
7 to 9 (TM1-4h, TM7-9h) and vertical TM 1 to 3 (TM1$3 v)$ must be sufficient to be able to distinguish between the bulk surface temperature of each TM tile $\left(\sim 55 \times 25 \mathrm{~mm}^{2}\right)$ and the radiant leading edges. Hence the incident angle of the convective plasma loads onto the divertor is very shallow $\left(<6^{\circ}\right)$, the incident convective flux on each leading edge is an order of magnitude higher than on the bulk of the tile surface. The remaining plasma facing surfaces of divertor and baffle are not intended to receive convective loads and are made of larger tiles $\left(\sim 100 \times 100 \mathrm{~mm}^{2}\right)$, so the leading edges are less critical and no resolution requirement is imposed. the Airy disc radius on the sensor must be limited to about 27,10 and $6 \mu \mathrm{m}$ for those points respectively.

The diffraction limit is defined by three critical apertures in the system, being the pinhole, the telescope and either the cold stop in the IR camera or the stop in the VIS objective lens. For most field points only the stop is limiting the beam diameter and the amount of light (besides obviously the blocking of light by the mirror M4 and its mounting struts in front of M3). Without consideration of tolerances, the optimum between the diffraction and aberration limit in the IR channel was

\begin{tabular}{|c|c|c|c|c|c|c|c|c|c|c|c|c|}
\hline & \multirow{2}{*}{ Pinhole } & \multicolumn{2}{|c|}{ Front mirrors } & \multicolumn{2}{|c|}{ Telescope } & \multirow{2}{*}{$\begin{array}{l}\text { Vacuum } \\
\text { Window }\end{array}$} & \multirow{2}{*}{$\begin{array}{l}\text { Beam } \\
\text { splitter }\end{array}$} & \multirow{2}{*}{$\begin{array}{l}\text { Corrector } \\
\text { lens }\end{array}$} & \multirow{2}{*}{ Objective lens } & \multicolumn{3}{|c|}{ Camera } \\
\hline & & M1 & M2 & M3 & M4 & & & & & Window & Cold filter & Sensor \\
\hline IR & 恸 & 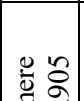 & 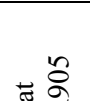 & ฮू ڤै & 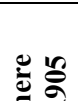 & $\approx$. & $\begin{array}{l}\text { Flat } \\
\text { Silicon } \\
4 \mu \mathrm{m}\end{array}$ & $\begin{array}{l}\text { Asphere } \\
\text { /flat } \\
\mathrm{Ge} \text { or Si }\end{array}$ & $\begin{array}{c}\text { Asphere/flat } \\
\text { Asphere/sphere } \\
\text { Both Ge or } \mathrm{Si}\end{array}$ & $\begin{array}{c}\text { Sapphire } \\
\text { flat }\end{array}$ & \begin{tabular}{|} 
Sapphire \\
$3.6-4.9 \mu \mathrm{m}$ \\
+ stop f/1.5
\end{tabular} & $\begin{array}{c}\text { InSb } \\
1280 \times 1024 \\
15 \mu \mathrm{m}\end{array}$ \\
\hline VIS & 琹 & 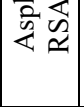 & 玒 芯 & $\begin{array}{l}\bar{z} \\
\frac{2}{4}\end{array}$ & 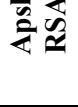 & II & $\begin{array}{c}\text { Flat } \\
\text { reflection }\end{array}$ & $\begin{array}{l}\text { Asphere } \\
\text { /flat } \\
\text { N-BK7 }\end{array}$ & $\begin{array}{c}\text { 6x sphere/sphere } \\
+ \text { stop f/2, 4xN- } \\
\text { BK11, F2HT, N-BK7 }\end{array}$ & & & $\begin{array}{c}\text { cMOS } \\
2560 \times 2160 \\
6.5 \mu \mathrm{m}\end{array}$ \\
\hline
\end{tabular}

To meet the design goals, a sequential Zemax model was developed, see Fig. 4 and Table 1. Each highly loaded divertor area is modelled with a separate configuration in which the divertor area is simplified to a best-fit flat surface (object surface in Zemax model). On each object surface five field points are defined, representing the four corners and the central point. To ensure the entire FOV passes through the optical system and fits onto the sensor, one additional configuration is made with a best-fit flat surface through the outermost points of the baffle area.
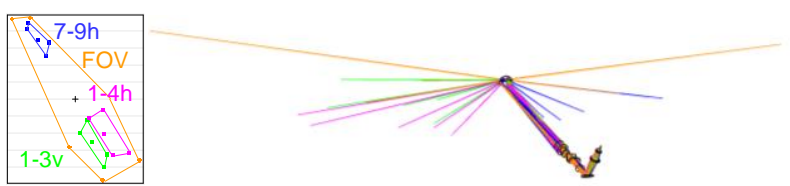

Fig. 4: Sequential Zemax model with 4 configurations: Footprint on sensor (left), lines of sight from divertor to sensor (right)

With the aim that the bulk temperature of each single tile is imaged onto at least one pixel of the IR camera without disturbance by radiation from the leading edge, at least 3 pixels per tile length are required and $15 \%$ MTF (Magnitude of the Optical Transfer Function) at a line pair width of half the pixel size. Projecting the divertor tile contours along the lines of sight onto the best-fit surface, the maximum allowed size on the best-fit surface that is imaged by one pixel, is calculated at $8 \mathrm{~mm}$ and the maximum line pair width at $15 \% \mathrm{MTF}$ is $4 \mathrm{~mm}$.

With a Python script, the magnification factor is calculated in two orthogonal directions from the object surface to the image surface for each field point to scale the pixel size and line pair width at $15 \%$ MTF (which is typically calculated at the image) onto the object surface. The distance of the field points to the pinhole varies Table 1Optical components of AEA endoscope. Pupils are marked bold between 1-3 m, resulting - after consideration of the angle between the line of sight and the surface normal - in a magnification factor ranging between 150 for the nearest points at TM3v and TM4h, and 400 to 700 for the outermost points at TM1v and TM9h, see Fig. 5. To meet the MTF criterion at $4 \mathrm{~mm}$ line pair width on the divertor, found to be an f-number of 1.2. However, aberrations increase significantly when tolerances are taken into account, resulting in a reduced optimum stop size of $\mathrm{f} / 1.5$. The resulting line pair width at $15 \%$ MTF is shown in Fig. 5 . The slight violation of the requirement in the tangential direction at the outermost points is accepted.

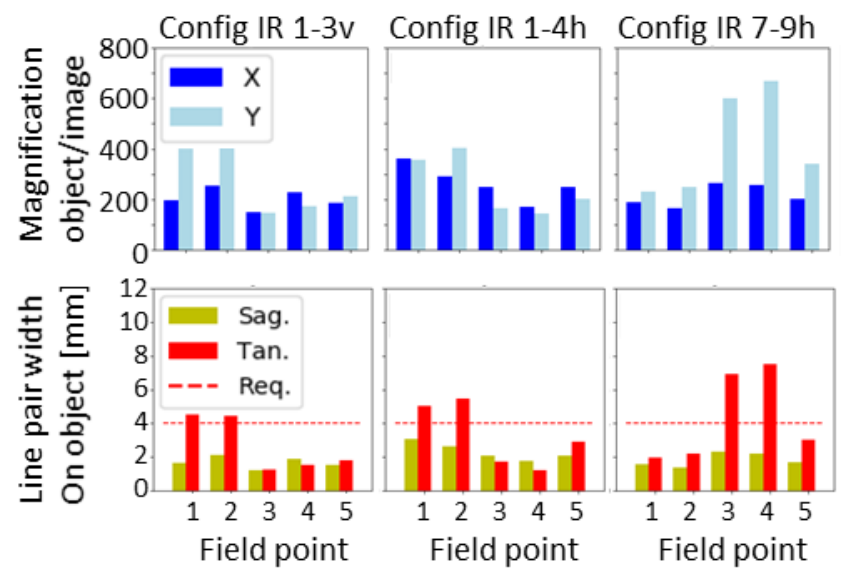

Fig. 5: Magnification factors for each field point from sensor to divertor (top) and line pair width at 15\% MTF (bottom)

\section{Thermo-mechanical analyses}

\subsection{Plasma loads}

The endoscope is not exposed to direct convective loads from the plasma, but fast ion losses of the neutral beam injection (NBI) typically hit the outer wall of the plasma facing components in the magnetic wells between the coils, i.e. at port openings. With the Ascot code, the areas where fast ions are deposited, were assessed for high mirror and standard magnetic field [1]. It appears that the

water-cooled head of the AEA endoscopes is not loaded, whereas the heads of the AEF endoscopes in upper side of the machine are loaded up to $1 \mathrm{MW} / \mathrm{m}^{2}$, see Fig. 6 . It requires protective measures that are not yet designed. 

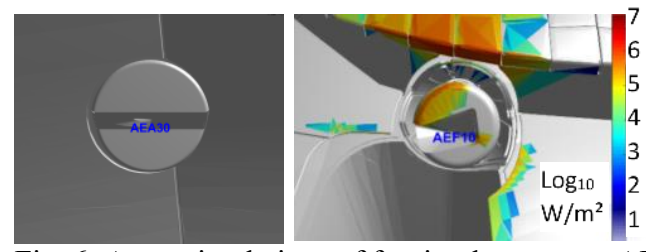

Fig. 6: Ascot simulations of fast ion losses onto AEA (left) and AEF endoscopes (right)

The radiative loads were calculated for all ports [5] based on the ray tracing method of [6] resulting in a design radiative heat flux onto the AEA (AEF) endoscopes of $102(56) \mathrm{kW} / \mathrm{m}^{2}$. In the thermal analyses, it was assumed that the plasma radiation is in the VUV range, so $100 \%$ is absorbed by the plasma exposed surfaces.

\subsection{ECRH stray radiation}

ECRH stray radiation, resulting from the microwave heating that is not absorbed by the plasma, is nearly fully reflected by all metal surfaces. It poses a risk for absorbing materials like ceramics and Kapton-insulated electrical cables inside the plug-in and optical box.

The ECRH is beamed into the plasma in module M1 and $\mathrm{M} 5$, resulting in attenuation from $110 \mathrm{~kW} / \mathrm{m}^{2}$ in $\mathrm{M} 1 / \mathrm{M} 5$ and $17 \mathrm{~kW} / \mathrm{m}^{2}$ in $\mathrm{M} 2 / \mathrm{M} 4$ to $3.5 \mathrm{~kW} / \mathrm{m}^{2}$ in $\mathrm{M} 3$.

To determine the stray radiation level inside the plugin, an FE simulation is made in Abaqus using cavity radiation [7] between all surfaces inside the plug-in. The absorption coefficients $\alpha$ of ECRH stray radiation are $\alpha_{S S}=1 \%$ for stainless steel, $\alpha_{R S A}=0.25 \%$ for RSA905 and $\alpha_{s a}=75 \%$ for the sapphire vacuum window [8] (actually $\sim 1 \%$ is absorption and rest is transmitted). To limit the size of the radiation matrix the cavity is split into four cavities separated by dummy surfaces with $100 \%$ absorption, see Fig. 7.

All surfaces are fixed at $0 \mathrm{~K}$ except the dummy surfaces. The dummy surface closing off the cutout of the head in front of the pinhole has a fixed temperature of $498 \mathrm{~K}$ corresponding to the incident heat flux of $3.5 \mathrm{~kW} / \mathrm{m}^{2}$, while the temperatures of the other dummy surfaces are free. The calculated heat flux absorbed by each surface, see Fig. 7, is exported to the thermal FE analyses as heat load.

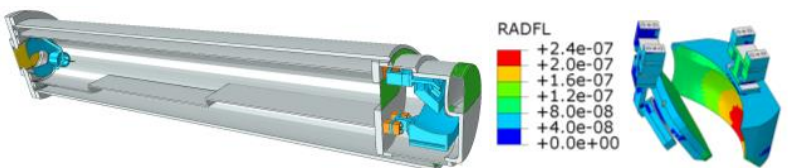

Fig. 7: ECRH stray radiation model with dummy surfaces in green (left) and resulting heat loads on front mirrors (right)

Inside the cavity of the head and the tube, the ECRH stray radiation level is $\sim 9$ and $\sim 2 \mathrm{~W} / \mathrm{m}^{2}$ respectively. The total load onto the sapphire window is $12.9 \mathrm{~mW}$ of which $12.7 \mathrm{~mW}$ is transmitted into the optical box $\left(Q_{\text {in }}\right)$ and $0.15 \mathrm{~mW}$ is absorbed, leading to a temperature rise $<1 \mathrm{~K}$ over a 30 minutes discharge (given a heat capacity of $0.152 \mathrm{~kg} \cdot 105 \mathrm{~J} / \mathrm{kgK}=16 \mathrm{~J} / \mathrm{K})$. The stray radiation intensity inside the optical box $q_{b o x}$ is estimated based on equilibrium between in- and outgoing radiation through the sapphire window with area $A_{w}$ and absorbed power on the $3 \mathrm{~m}^{2}$ surface area of the stainless steel box $A_{b o x}$, i.e. $q_{b o x}=Q_{\text {in }} /\left(A_{w} \alpha_{s a}+A_{b o x} \alpha_{s s}\right)=342 \mathrm{~mW} / \mathrm{m}^{2}$.

It leads to a radiant equilibrium temperature $<21{ }^{\circ} \mathrm{C}$ for an object that absorbs all ECRH stray radiation. Clearly, the stray radiation level inside the optical box is acceptable for all sensitive electronic components.

\subsection{Thermo-mechanical analyses}

Heat loads are the radiative load on the plasma facing surfaces of the head and the pinhole as well as the ECRH stray radiation on all plug-in surfaces as exported from the calculations of the previous section. As for the ECRH stray radiation, the radiation inside the plug-in is modelled with four cavities, this time using an emissivity for infrared light of $0.7,0.3,0.1$ and 0.01 for thermal insulation, steel, RSA905, and sapphire, respectively.

The total radiation load onto the plasma exposed surface of the plug-in of $53 \cdot 10^{-3} \mathrm{~m}^{2}$ is $5.3 \mathrm{~kW}$. The head is supplied with a maximum water flow of $0.2 \mathrm{l} / \mathrm{s}$ (heat capacity of water $=4.2 \mathrm{~kJ} / \mathrm{kgK}$ ), resulting in temperature increase of $5.3 / 0.2 / 4.2=6.3 \mathrm{~K}$. In the thermal analyses of the plug-in, the internal surface of the head is simply fixed at $20{ }^{\circ} \mathrm{C}$. (The detailed thermal response of the head is presented in chapter 6).

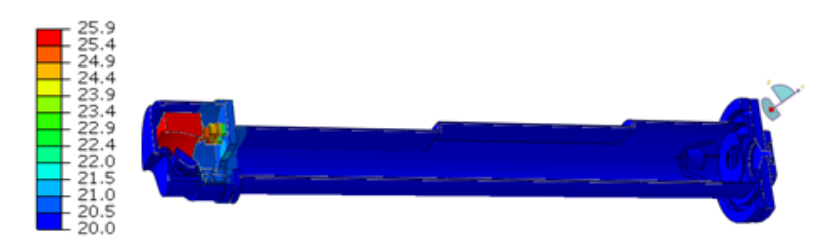

Fig. 8: Temperature profile after $1800 \mathrm{~s}$ of plasma operation

In Fig. 8 the thermal response is shown. The most highly loaded component is front mirror M1. Mirror M1 and M2 are thermally insulated to minimize thermal losses during the heating cycle. Mirror M1 is made as massive as space constraints allow, to limit the temperature rise during plasma operation. The temperature rise inside $\mathrm{M} 1$ reaches $6.2 \mathrm{~K}$ and the gradient inside M1 is limited to $0.36 \mathrm{~K}$. The temperature rise causes a change of the radius of the optical surface of $\sim 0.01 \%$, It causes a maximum relative reduction of the MTF over all field points at the Nyquist frequency of the IR camera, i.e. a line pair width of $30 \mu \mathrm{m}$ of only $1.5 \%$. The temperature rise in the telescope mirrors M3/M4 remains below $0.5 \mathrm{~K}$. Despite this small amount, the maximum relative reduction of the MTF increases to $2.7 \%$. The effect can be counteracted by refocusing the IR camera, which is realized by the remotely controlled linear motor.

During the heating cycle of the front mirrors, no irreversible deformations should occur inside the mirrors. For stress released RSA905 with a Young's modulus $E$ of $90 \mathrm{GPa}$, thermal expansion coefficient $\alpha_{T}$ of $19 \mu \mathrm{m} / \mathrm{mK}$ and a yield limit $R_{0.2}$ of $100 \mathrm{MPa}$ at $350{ }^{\circ} \mathrm{C}$, no plastic strains can occur up to an internal temperature gradient of $R_{0.2} / E / \alpha_{T}=60 \mathrm{~K}$. It has been calculated that for heating from 20 to of $350{ }^{\circ} \mathrm{C}$ in 2 hours, the maximum temperature gradient is $20 \mathrm{~K}$ in $\mathrm{M} 1$ and $13 \mathrm{~K}$ in $\mathrm{M} 2$, i.e. safely below the limit. The required heating power is $135 \mathrm{~W}$ for M1. Since the mirrors are insulated, they 
mainly cool down due to radiation, which takes about 12 hours after turning off the heating.

The pre-stress in the mounting bolts of M1 and M2 must be preserved over the heating cycles. The insulated support structure of each mirror is fixed with four M5 bolts with a spring washer. The insulation is Dotherm $500 \mathrm{M}$ which successfully passed the outgassing test. With a pre-stress of $250 \mathrm{MPa}$, the peak stresses over the heating cycle in the insulation remain within the strength limits and the stress in the bolts drops only by $10 \mathrm{MPa}$ during the cycle. So there is no risk of loss of pre-stress.

Finally, the deformation of the plug-in due to gravity was assessed. The maximum displacement of the front mirrors relative to the telescope mirrors is $0.04 \mathrm{~mm}$.

\section{Tolerances}

\subsection{Assembly and installation strategy}

The assembly strategy is to purchase all optical components from industry including a stand-alone verification of the optical performance and to assemble them into the endoscope in the IPP laboratory. The telescope mirrors M3/M4, the IR and VIS objective lens and the beam splitter with the IR corrector lens are all purchased as pre-assembled units. The other optical components are bought as stand-alone units. The supplier has to measure the geometrical relation between the optical coordinate system of the unit and conical cutouts on the outside of the unit that are used as reference marks during assembly.

The assembly of the endoscope starts with mounting the telescope onto the vacuum flange that is fixed vertically to the assembly frame. All optical units will be accurately positioned relative to the telescope with the aid of a simple adjustment part. After initial mounting of the unit, the target shape of the adjustment part is determined by measurement of the reference mark positions of the corresponding unit. After remachining of the adjustment part, the unit is remounted. Fitting pins must ensure that the positioning of the adjustment part during the position measurements is highly repeatable. With this approach, no tolerance requirement needs to be imposed onto the support tube of the plug-in nor the optical base plate of the optical box. Moreover, permanent deformation due to gravity is cancelled out by the adjustment parts. After assembly, the optical box is dismounted from the vacuum flange to allow for a separate installation of the plug-in and optical box onto W7-X. As before, fitting pins ensure that the original position of the optical box is precisely restored after installation.

The tilt of front mirror M2 is manually adjusted to compensate for position errors of the front mirrors by centering the central ray onto the primary telescope mirror M3. As final step, positon of the IR and VIS camera will be adjusted to find the optimum optical performance.

\subsection{Tolerance assessment}

The following errors were considered: power and irregularity errors on each optical surface; refraction index error, thickness and wedge deviations of each lens; relative position and tilt errors of components inside preassembled units, between units and between plug-in and optical box. In total 130 tolerances were defined. The allowed magnitude of each tolerance was determined in a sensitivity study. The increase of the spot size radius due to a single tolerance was limited to $\sim 5 \%$ while the tilt of M2 and the camera position are used as compensators. It resulted in an allowed magnitude of $30-600 \mathrm{~nm}$ for surface errors, $5-20 \mu \mathrm{m}$ for relative position errors inside units and 0.1-1 mm for relative position errors between units. Suppliers confirmed the feasibility of these limits.

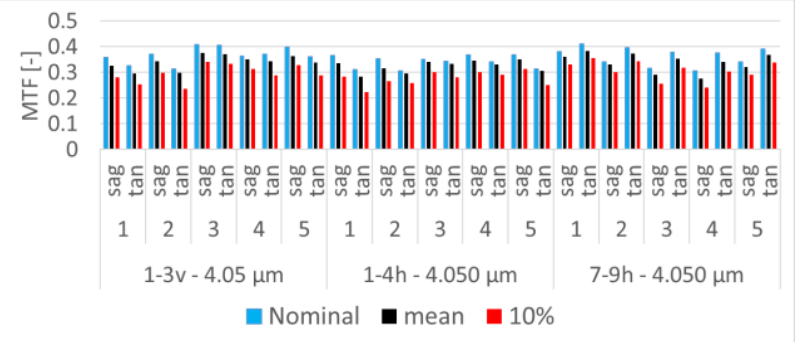

Fig. 9: Reduction of MTF due to tolerances

With 100 Monte Carlo (MC) simulations, the effect on the MTF of a random distribution of the tolerances within the predefined limits was assessed. At the Nyquist frequency of the IR camera, the worst $10 \%$ of the MC results still reaches an MTF of $20 \%$ for all field points, see Error! Reference source not found., which is acceptable.

\section{3D-printed water-cooled head}

The complex shape of the water-cooled head requires the flexible manufacturing technique of $3 \mathrm{D}$ printing, or more specifically: selective laser melting (SLM) of steel powder (voxel size $\sim 50 \mu \mathrm{m}$ ) in an airtight chamber under protective gas. With this technique, the head is printed layer by layer. Circular internal channels without intermediate supports are feasible up to $10 \mathrm{~mm}$ diameter. Six parallel channels of 3.4 to $3.5 \mathrm{~mm}$ are chosen within the wall thickness of $8 \mathrm{~mm}$. The summed cross section of the channels matches that of the supply line of $\varnothing 10 \mathrm{~mm}$.

Samples of $100 \mathrm{~mm} \varnothing 10 \mathrm{x} 1$ pipes of EN 1.4404 have been used to qualify the material for W7-X, demonstrating a low Cobalt content of $290 \mathrm{ppm}$, a magnetic permeability $<1.01$, good weldability and passing a pressure test up to the testing limit of 1000 bar.

According to hand estimations using the Moody diagram by simplifying the channels to straight tubes, the pressure loss at $0.2 \mathrm{l} / \mathrm{s}$ is $1.1 \mathrm{bar}$ in the head and 3.4 bar due to the central ring line, which is well below the W7-X requirement of max. $6 \mathrm{bar}$ pressure loss. With computational fluid dynamics (CFD) simulations in Abaqus, the flow speed and pressure loss was calculated, see Fig. 10. The calculated pressure loss was 1.3 bar. Finally, a full scale prototype was manufactured in AlSi10Mg to verify the pressure loss and equal flow distribution, see Fig. 10. The measured pressure loss is 2 bar, still well within the W7-X specification.

The temperature of the head remains generally well below $100{ }^{\circ} \mathrm{C}$ with small peaks of $\sim 200{ }^{\circ} \mathrm{C}$ in steady state, 
see Fig. 10, leading to equivalent stresses $<120 \mathrm{MPa}$ due to water pressure alone and $<300 \mathrm{MPa}$ including heating.

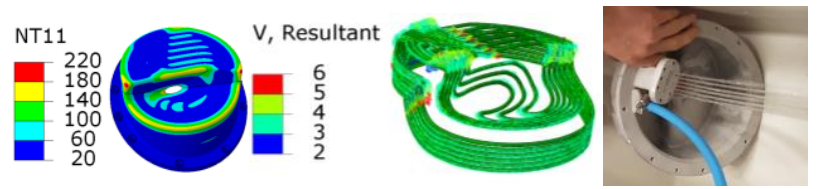

Fig. 10: Water-cooled head model (left), flow velocity (middle) and laboratory test (right)

\section{Conclusions}

The optical design of the steady state compatible endoscopes for the divertor monitoring of W7-X was completed including the thermo-mechanical confirmation of the design. The optical components are being purchased from industry and auxiliary components are being detailed. Next steps are acceptance tests of the optical performance of units, the assembly of the units into the endoscope and the verification of the overall optical performance in the laboratory.

\section{Acknowledgments}

This work has been carried out within the framework of the EUROfusion Consortium and has received funding from the Euratom research and training programme 20142018 and 2019-2020 under grant agreement No 633053. The views and opinions expressed herein do not necessarily reflect those of the European Commission.

\section{References}

[1] D. Chauvin, et al. Design and manufacturing progress of IRVIS endoscopes prototypes for W7-X divertor temperature monitoring http://www.euro-fusionscipub.org /wp-content/uploads/WPS1CP16 16617 submitted.pdf

[2] Jakubowski et al. Infrared imaging systems for wall protection in the W7-X stellarator, Review of Scientific Instruments 89, 10E116 (2018); https://doi.org/ $10.1063 / 1.5038634$

[3] Wurden et al. A divertor scraper observation system for the Wendelstein 7-X stellarator, Review of Scientific Instruments 89, 10E102 (2018); https://doi.org/10.1063/ $\underline{1.5035078}$

[4] S. Äkäslompolo et.al., Armoring of the Wendelstein 7-X divertor-observation immersion-tubes based on NBI fastion simulations, Fusion Engineering and Design 146, Part A, (2019), pp. 862-865 https://doi.org/10.1016/ j.fusengdes.2019.01.099

[5] A. Carls et al., Numerical design approval of W7-X port liners, Fusion Engineering and Design, Volume 146, Part B, September 2019, Pages 2761-2764 https://doi.org/ $\underline{10.1016 / j . f u s e n g d e s .2019 .05 .027}$

[6] Eich, T., \& Werner, A., Numerical studies on radiative heat loads to plasma-facing components for the W7-X stellarator. Fusion Science and Technology, 53(3), (2008) pp. 761-779.

[7] Abaqus user's manual, Theory guide, section 2.11.4 (2016)

[8] F. Leipold et al., Polarizer design for millimeter-wave plasma diagnostics, Review of Scientific Instruments 84(8), 2013 https://aip.scitation.org/doi/10.1063/1.4816724 